\title{
POSTURAL EVALUATION OF PATIENTS WITH TEMPOROMANDIBULAR DISORDERS UNDER USE OF OCCLUSAL SPLINTS
}

Paulinne Junqueira Silva Andresen STRINI ${ }^{1}$, Naila Aparecida de Godoi MACHADO ${ }^{2}$, Marília Cavalheri GORRERI ${ }^{3}$, Amanda de Freitas FERREIRA ${ }^{4}$, Gilmar da Cunha SOUSA ${ }^{5}$, Alfredo Júlio FERNANDES NETO ${ }^{6}$

\author{
1- MSc, Dental School, Federal University of Uberlândia, Uberlândia, MG, Brazil. \\ 2- MSc, PhD student, Dental School, Federal University of Uberlândia, Uberlândia, MG, Brazil. \\ 3- MSc in Physical Therapy and Trauma Orthopedics, University Center of Triângulo, Uberlândia, MG, Brazil. \\ 4- Undergraduate student, Dental School, Federal University of Uberlândia, Uberlândia, MG, Brazil. \\ 5- PhD, Professor, Institute of Biomedical Sciences, Area Morphology, Federal University of Uberlândia, Uberlândia, MG, Brazil. \\ 6- PhD, Professor, Dental School, Federal University of Uberlandia, Uberlândia, MG, Brazil.
}

Corresponding address: Prof. Dr. Alfredo Júlio Fernandes Neto - Av. Pará, 1720. Campus Umuarama, - Bl 2B, Sala 2B01, 38400-902 - Uberlândia/MG

- Phone: +55 34 3218-2222 - e-mail: alfredon@ufu.br

Received: June 20, 2008 - Modification: July 08, 2008 - Accepted: January 10, 2009

\begin{abstract}
$O$ bjectives: Alterations in the temporomandibular complex can reflect in adaptations of the individual's entire muscular system, intervening with the head position and scapular waist, developing postural alterations and modifying all corporal biomechanics. The aim of this study was to evaluate the head position (HP) and head postural alterations before and after installation of occlusal splints. Material and Methods: Twenty patients with temporomandibular disorders (TMD) underwent clinical and postural examination, before the installation of an occlusal splint, and after 1 week and 1 month of use. Results: There were statistically differences for HP, between the initial values and after 1 week of use of the occlusal device ( $p=0.048)$ and also between 1 week and 1 month of evaluation $(p=0.001)$. Decrease of the painful symptomatology and maintenance of the rectification were also observed. Conclusions: The individual's postural position can suffer biomechanical alterations due to stomatognathic alterations, causing clinically visible changes in dysfunctional individuals and affecting the performance of the involved structures.
\end{abstract}

Key words: Temporomandibular disorders. Postural alterations. Occlusal splint.

\section{INTRODUCTION}

Temporomandibular disorders (TMD) is a collective term which refers to a large number of clinical problems that involve the masticatory musculature, temporomandibular joint (TMJ) or both and the associated structure ${ }^{25}$. TMD etiology is multifactorial. It may be related to an imbalance among occlusal, anatomical, psychological and neuromuscular factors, providing neck and head structural dysfunction ${ }^{3}$.

The TMJ is directly related to the cervical and the scapular region by an interrelated neuromuscular system. Changes in the cervical spine can cause TMJ disorders and the opposite is also true. Since head and cervical muscles are closely related to the stomatognathic system, studies have been carried out to confirm that postural changes of the head and the body could have an adverse biomechanical effect on the TMJ and lead to TMD ${ }^{1,20}$.

The head position seems to affect the position of the mandible. Furthermore, the masticatory muscles that are related to the neck and trunk can also be affected. These muscles are susceptible to suffer the direct influence of the force of gravity. Changes in the head posture in the sagittal plane result in alterations in the pattern of habitual mouth closing and also in the patient ability to reach the intercuspation position ${ }^{19}$. Gonzalez and Manns ${ }^{7}$ (1996) showed that the anterior head position is accompanied by changes in mandible positioning, with a decrease of the physiological space, which occurs as a result of the displacement of the mandible upwards and backwards due to excessive contraction of the masticatory muscles ${ }^{7}$.

The neck muscles are essential to maintain the balance of the head and the muscles of the stomatognathic system, which could be seen as a coordinated system, in which an intervention at any level could result in changes in this complex. In this way, changes induced in the head posture cause alterations in the activity of the masticatory muscles. Therefore, the manipulation of the muscles of the mandible results in alterations in the usual head posture?

The additional compensation mechanism attributed to 
the cervical region after an anterior posture of the head can alter the masticatory system and, therefore, the patients become more susceptible to stress, pain and muscular spasms. The abnormal head position refers to the most common manner of bad posture and is the cause of many painful myofascial disorders ${ }^{30}$. The aim of this study was to evaluate the head position (HP) and the painful symptomatology before and after the installation of occlusal splints.

\section{MATERIAL AND METHODS}

\section{Subjects}

Twenty patients with TMD (19 female and 1 male) aged $17-43$ (mean age $=28.4 \pm 8.4$ years) enrolled in the Program for Reception, Treatment and Monitoring of Patients with TMD and Orofacial Pain of the Dental School of Federal University of Uberlândia (FOUFU) were evaluated.

A number of inclusion criteria were considered. The sample was consisted of patients with mixed TMD ${ }^{10}$ and parafunctional habits. The patients complained of muscular pain, articular sounds, headache and dental clenching. The signs and symptoms should be present for a minimum period of 6 months, indicating chronic pain ${ }^{18}$. At least 20 functional teeth should be present in the oral cavity in order to allow for bilateral occlusion, which is a condition that provides an adequate occlusal stability. All eligible patients showed sleep bruxism with indication for use of an occlusal splint. As the use of occlusal splints in control subjects is not indicated because of the absence of signs and symptoms of TMD and ethical implications, this study evaluated the alterations occurred in patients with TMD before and after stomatognathic alterations promoted by the installation of occlusal splints.

The following exclusion criteria were considered for patients not to enter the study: no painful symptoms and muscular involvement, Class III Angle occlusion, indication for or ongoing orthodontic treatment, history of facial and TMJ trauma, history of TMJ dislodgment, need for treatment or extensive dental rehabilitation, systemic medical conditions, such as rheumatic, hormonal, infectious, nutritional and metabolic disorders, history of systemic diseases that might jeopardize the synovial organic joints, including the TMJs, biomechanical common predisposing factors, including skeletal malformations and postural alterations.

\section{Procedures}

Prior to initiation of the study, the research protocol was approved by the Ethics Committee of the Federal University of Uberlândia (protocol n. 423/06) and all subjects signed an informed consent form after receiving full explanation of the study nature and purposes.

Patients were diagnosed with mixed TMD and the presence of parafunctional habits, such as sleep bruxism, was identified by clinical interview and examination. Palpation was carried out on the TMJ and masticatory and cervical muscles ${ }^{26}$. Any report of pain, discomfort, or sign or symptom of dysfunction was duly recorded and these rates were used for diagnosed TMD. The data collected in this step was sufficient for fulfilling the American Association of Orofacial Pain (AAOP) criteria $^{15}$ and identify the presence of TMD. To measure the level of pain, the Visual Analog Scale (VAS) was used at the beginning of the study and 1 week and 1 month after use of the occlusal splint ${ }^{27}$. Mild pain was considered in VAS when the patient made the pencil mark between 1 and $33 \mathrm{~mm}$; moderate pain when the mark was made between 34 and $66 \mathrm{~mm}$ and severe pain was considered when the mark was made between 67 and $100 \mathrm{~mm}^{27}$.

The assessment of posture was held with the patient standing on a smooth surface, in a relaxed position, without shoes and with his/her feet in the same width of shoulders, in a frontal and natural head position. The HP was obtained by placing a millimeter ruler vertically from the rear portion of the skull to the thoracic spine. Since then, the horizontal distance of the cervical spine by that line of vertical bob was also measured with the ruler (Figure 1). The normal distance ranges from 6 to $8 \mathrm{~cm}^{26}$. This physical examination was done with the aid of a physical therapist, duly trained and authorized to implement the procedures. The physical therapist was blinded to the measurements done in the

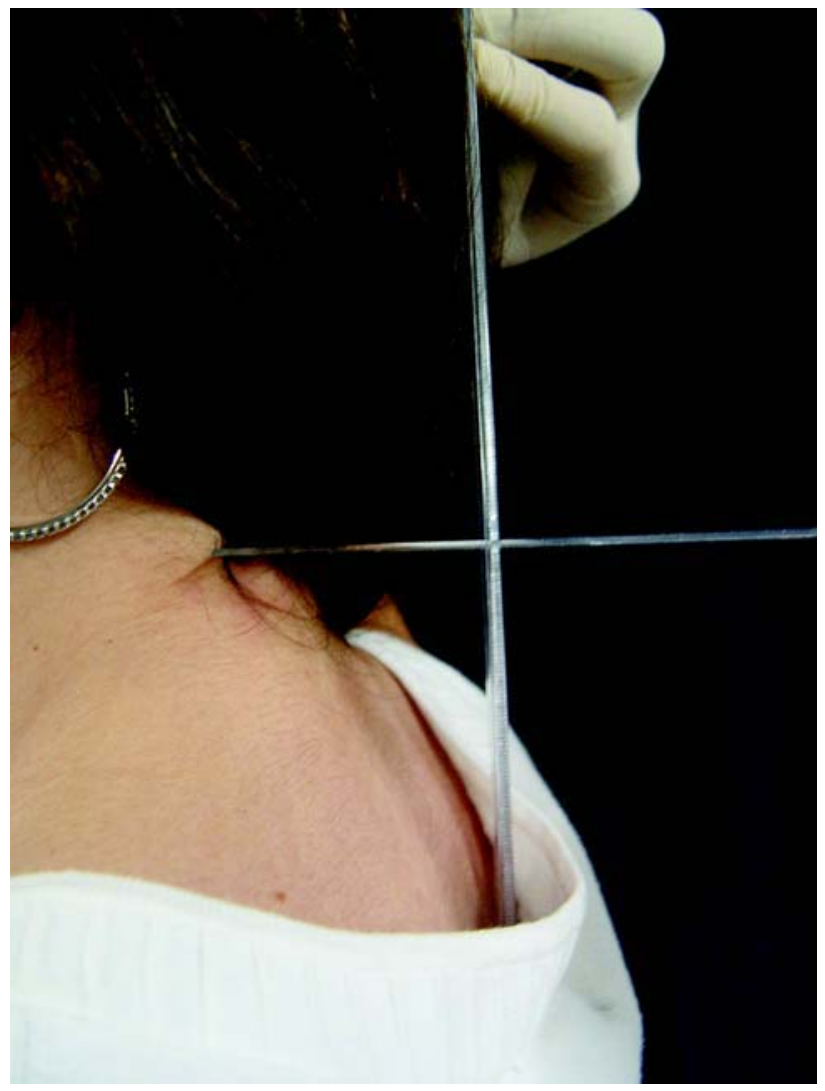

FIGURE 1- Lateral left view of head position (HP) measurement procedure by placing a millimeter ruler vertically in the rear portion of the skull to the thoracic spine and another one measuring the horizontal distance of the cervical spine and the line of vertical bob 
patients in each step of this study.

After the subjective, clinical and physical evaluations of the patients had been completed, the next step was to prepare occlusal splints, following the principles established by Okeson $^{21}$. Alginate impressions were taken and the cast models were obtained. The upper model of each patient was cut and vacuum pressed in a 2-mm acetate sheet in order to obtain the occlusal splint. After trimming the borders, the occlusal splints were relined with acrylic resin. This could allow the occlusal adjustment in centric relation ${ }^{21}$.

For each patient, an occlusal splint was fabricated programming the mandibular position in centric relation occlusion (CRO), occlusal stability and anterior guidance. The occlusal splint was adjusted according to the parameters of an organic occlusion: the occlusal forces should be directed to the long axis of the posterior teeth, over their occlusal faces, eliminating any contact among upper and lower anterior teeth (and the opposite also be true) $)^{21}$. The patients were instructed to use the splint continuously during $24 \mathrm{~h}$, removing it only for eating and cleaning, within the first week of treatment. After this period, the patients were instructed to use the splint at night, being free to use it during the day in case of pain.

The follow-up visits were scheduled to occur after 1 week and after 1 month of use, and the occlusal splints were reviewed and adjusted where and when necessary. A quick review of the history and main complaint was also carried out to specify whether there was any improvement of the symptoms. A new clinical examination and the analysis of the physical posture of the patients could also be performed.

The placement of an occlusal device is usually the starting point for treatment. Since therapy with devices is directed at only one aspect of the problem, it has to be used in combination with other therapeutic modalities, such as physical therapy, pharmacotherapy, behavioral modification and psychological counseling for the treatment to be effective ${ }^{26}$. As this study aimed at evaluating only the influence of a therapeutic modality, that is, the use of occlusal splint, the other arrangements were not considered in order to avoid interferences in the study outcomes.

\section{Statistical Analysis}

In order to determine whether there were statistically significant differences among the values of the previous position and of that obtained by the patients at the beginning, after 1 week and 1 month, Wilcoxon's rank sum test was performed. The level of significance was set at 0.05 , in a bilateral proof.

\section{RESULTS}

There was a significant reduction in the level of pain reported by the patients. Initially, $35 \%$ of the volunteers had severe pain, $40 \%$ moderate and $25 \%$ mild. After 1 week of use of occlusal splint for $24 \mathrm{~h}, 5 \%$ of patients had severe pain, $15 \%$ moderate, $75 \%$ mild and $5 \%$ no pain. After 1 month, $5 \%$ of the volunteers had severe pain, $20 \%$ moderate,
TABLE 1- Probability found, when applying the Wilcoxon's rank sum test of the values of head position obtained at the beginning of the study and after 1 week and 1 month of occlusal splint use

\begin{tabular}{ll}
\hline $\begin{array}{l}\text { Variables analyzed } \\
\text { HP }\end{array}$ & Probability \\
\hline Initial x week & $0.048^{*}$ \\
Initial x months & 0.206 \\
Week x months & $0.001^{*}$ \\
\hline
\end{tabular}

$\left({ }^{*}\right) p<0.05$.

$70 \%$ mild and $5 \%$ had no pain.

When analyzing the data concerning the clinical distance of HP, all patients $(100 \%)$ considerably lower values of reference, which may indicate a tendency to rectification of neck and head position, i.e., none of the patients in any stage of this research showed a posture of the head into the standards of normality or with anterior posture. However, it was observed that after 1 week of continuous use of the splint, $90 \%$ of patients tended to bring those values closer to the ideal conditions, and, after 1 month, this percentage dropped to $85 \%$, probably due to decrease in the time of use. Still regarding physical examination and the patient's posture, the values obtained in the HP at the start of treatment, after 1 week and 1 month were compared to each other. The results of this comparison are shown in Table 1. There were statistically significant differences $(p<0.05)$ among the values obtained at the beginning, after 1 week and after 1 month were found. When the variables were compared between the beginning and after 1 week, the latter period had higher values. Comparing the results obtained after 1 week and after 1 month, the earlier period had higher values.

\section{DISCUSSION}

The results of this study showed a higher prevalence of TMD in female patients, mainly in 2 th and 3 th decades of life. This greater involvement observed in women is consistent with the results of several studies ${ }^{17,21,22,26}$, which showed a ratio of 4 or 6 women to 1 man among all people seeking treatment, especially those between 2 nd and 4 th decades of life ${ }^{17}$.

The occlusal splint used in this study was consistent with the technique advocated by Okeson ${ }^{21}$ (1998). This kind of device is used in many cases of TMD, with significant results in relieving symptoms, myorelaxation and reduction of muscle hyperactivity. The acetate is a rigid material that allows a technique of making the splint quickly and easily, with low-cost (accessible to the patients), allowing the relining and reducing the number of clinical appointments to complete the device. The maximum thickness is selected according to the free space. 
The occlusal splint is considered a diagnostic device, assisting the professional management of patients with TMD. This device creates a reversible condition and is also effective in order to relieve the pain in neuromuscular deprogramming and consequently, in muscle relaxation, reducing hyperactivity of the masticatory muscles ${ }^{2,5,6,8,12,28}$. It is necessary to obtain a number of dental occlusal contacts, bilateral, uniform and symmetrically distributed. The splint quality is utterly related to the presence of excursion guides, good fitting, stability and retention, and smooth and polished surfaces.

In view of the multifactorial etiology of TMD, there are controversies concerning the influence of occlusal splint in reducing painful symptoms in patients with TMD. However, recent studies ${ }^{21,26,28}$ have shown that its use is valid and rational especially in cases of bruxism. In the present study, according to the measurement obtained by the Visual Analog Scale, there was a significant improvement on the pain reported by the patients following the use of the occlusal splint. A previous study that evaluated the effectiveness of occlusal devices also showed a significant reduction of pain $^{22}$, which may be related to changes in the occlusal condition, with temporary alterations in the position of previous dental contacts, liberating the masticatory system of occlusal disharmony and hence reducing the hyperactivity of the mandibular muscles.

Patients with TMD have a higher prevalence of cervical dysfunction, and the neck muscle activity can influence the activity of the masticatory muscles. This fact can be caused by an inadequate head position, which requires an additional demand on the cervical region, altering the entire masticatory system, increasing susceptibility to stress, pain and spasm ${ }^{30}$. All patients presented signals of psychological factors and symptoms irradiated for cervical and scapular region.

Landulpho, et al. ${ }^{13}$ (2004) reported that the device placed between the dental arches interrupts the proprioceptive information of mechanoreceptors located within the periodontal ligament enabling the muscles to return to activity and consequently to an improved balanced mandible position. In this study, the installation of an occlusal splint in centric relation contributed for a balanced muscle position and relieve of dysfunction.

The anterior head position or abnormal head position is considered one of the factors that may lead to the development of TMD, increased tension in the muscles and action of masticatory forces on the mandible ${ }^{19}$. The HP was clinically evaluated in this study, initially, after 1 week and after 1 month of treatment. Most patients showed a lower HP in the follow-up period, indicating a tendency for adjustment to the cervical region. In the present study, higher scores than those considered normal were observed at any of the periods, indicating that there was no head anteriorization in the sample with TMD. After the treatment, a small percentage tended to set aligned with an ideal position.

Huggare and Raustia ${ }^{9}$ (1992) observed, a significant straightening of the curvature of the spine after the occlusal therapy. Santander, et al. ${ }^{24}$ (1994) also reported that the insertion of the device caused an extension of the head and upper cervical spine and reduced the cervical spine lordosis. However, some studies ${ }^{1,20}$ have reported an increase in cervical lordosis in patients with TMD, not related to a leveling of the shoulders, which can lead to an abnormal head position. The occlusal adjustment is capable of changing the head posture, as shown in this study.

In the statistical analysis of HP results, the data from the beginning of the study, 1 week and 1 month of treatment were compared. Statistically significant differences among the HP values could be seen when the baseline data were compared to those obtained at 1 week and 1 month. The anterior head position was higher after 1 week of treatment with the use of the splint. Within 7 days of monitoring, there was an increase in the curvature of the cervical spine, tending to increase the HP close to the levels considered as a reference. The occlusal adjustment and elimination of occlusal interferences allowed an increase in cervical lordosis. As the frequency of use of the occlusal device decreased, there was a reduction in the HP values, and again a tendency to adjustment.

An extension of the head was observed after $1 \mathrm{~h}$ of opening mouth trial, showing a tendency to recover the original position of the head after removing the device ${ }^{4}$. In this study, a higher value of HP was observed after 1 week and the decline of this value after 1 month can only be due to the use of the occlusal splint at night, without the patient using the device for several hours during the day. The removal of the occlusal plate inhibits the effects obtained and causes a return to original condition ${ }^{11}$.

Postural alterations are related to changes of position in the head segment, neck and cervical spine, conditions that are considered to be abnormalities of the normal standard posture $^{11}$. From the structural point of view, there is a significant correlation between the position of head and the craniofacial and dental-alveolar morphologies ${ }^{16,23,29}$. Lee, Okeson and Lindroth ${ }^{14}$ (1995) stated that correct posture is particularly associated with health status and any alteration on this posture could lead to pain and dysfunction of the craniocervical system. They suggest that the head posture could elucidate some symptoms of TMD and, hence, that the clinical correctness of the position of the head can reduce these symptoms. It is clear that associated factors are present in patients under these conditions, being necessary a multidisciplinary team to treat these disorders.

All this rearrangement of body structures set served in order to seek a return of physiological conditions ideal for the body. The range of changes studied and the effects caused by the alteration of the occlusal condition can affect various components and body structures that are interconnected. Thus, it is essential that further research is done in order to understand the interaction between TMD and the structures associated with the adjacent tissue located at a distance. 


\section{CONCLUSION}

The results of the present study suggest an important interrelation between occlusion, TMD, head position and pain. In conclusion, the postural position of the individual can suffer biomechanical alterations originated from stomatognathic modifications in dysfunctional individuals, causing clinically visible alterations and affecting the performance of the involved structures.

\section{ACKNOWLEDGMENTS}

The authors are grateful to FAPEMIG to financial support (CDS 1359/05) and Prof. Roberto Bernardino Júnior, Msc, for his contribution in the development of this study.

\section{REFERENCES}

1- Amantéa DV, Novaes AP, Campolongo GD, Barros TP de. The importance of the postural evaluation in patients with temporomandibular joint dysfunction. Acta Ortop Bras. 2004;12(3):155-9.

2- Chandu A, Suvinen TI, Reade PC, Borromeo GL. The effect of an interocclusal appliance on bite force and masseter electromyography in asymptomatic subjects and patients with temporomandibular pain and dysfunction. J Oral Rehabil. 2004;31(6):530-7.

3- Clark GT, Green EM, Dornan MR, Flack VF. Craniocervical dysfunction levels in a patient sample from a temporomandibular joint clinic. J Am Dent Assoc. 1987;115(2):251-6.

4- Daly P, Preston CB, Evans WG. Postural response of the head to bite opening in adult males. Am J Orthod. 1982;82(2):157-60.

5- Dawson PE. A classification system for occlusions that relates maximal intercuspation to the position and condition of the temporomandibular joints. J Prosthet Dent. 1996;75(1):60-6.

6- Dawson PE. Centric relation. Its effect on occluso-muscle harmony. Dent Clin North Am. 1979;23(2):169-80.

7- Gonzales HE, Manns A. Forward head posture: its structural and functional influence on the stomatognathic system; a conceptual study. Cranio. 1996;14(1):71-80.

8- Hiyama S, ONO T, Ishiwata Y, Kato Y, Kuroda T. First night effect of an interocclusal appliance on nocturnal masticatory muscle activity. J Oral Rehabil. 2003;30(2):139-45.

9- Huggare JA, Raustia AM. Head posture and craniovertebral and craniofacial morphology in patients with craniomandibular dysfunction. Cranio. 1992;10(3):173-9.

10- IASP. Classification of chronic pain: descriptors of chronic pain syndromes and definitions of pain terms. 2nd ed. Seattle: IASP Press; 1994.

11- Kibana Y, Ishijima T, Hirai T. Occlusal support and head posture. J Oral Rehabil. 2002;29(1):58-63.

12- Kovaleski WC, De Boever J. Influence of occlusal splints on jaw position and musculature in patients with temporomandibular joint dysfunction. J Prosthet Dent. 1975;33(3):321-7.
13- Landulpho AB, Silva WAB, Silva FA, Vitti M. Electromyographic evaluation of masseter and anterior temporalis muscles in patients with temporomandibular disorders following interocclusal appliance treatment. J Oral Rehabil. 2004;31(2):95-8.

14- Lee WY, Okeson JP, Lindroth J. The relationship between forward head posture and temporomandibular disorders. J Orofac Pain. 1995;9(2):161-7.

15- Leeuw R. Post MVA TMD patients endorse more general symptoms than nontrauma TMD patients. J Evid Based Dent Pract. 2008;8(4):246-

16- Marcotte MR. Head posture and dentofacial proportions. Angle Orthod. 1981;51(3):208-13

17- McNeill C. Management of temporomandibular disorders: concepts and controversies. J Prosthet Dent. 1997;77(5):510-22.

18- McNeill C, Dubner R. What is pain and how do we classify orofacial pain? In: Lund JP, Lavigne GJ, Dubner R, Sessle BJ, editors. Orofacial pain: from basic science to clinical management. Quintessence Publishing, Carol Stream; 2001.

19- Mohl ND. Head posture and ist role in occlusion. N Y State Dent J. $1976 ; 42(1): 17-23$.

20- Nicolakis P, Nicolakis M, Piehslinger E, Ebenbichler G, Vachuda M, Kirtley C, Fialka-Moser V. Relationship between craniomandibular disorders and poor posture. Cranio. 2000;18(2):106-12.

21- Okeson JP, editor. Management of temporomandibular disorders and occlusion. 4th ed. St. Louis: Mosby; 1998.

22- Okeson JP, Moody PM. Evaluation of occlusal splint therapy and relaxation procedures in patients with temporomandibular disorders. J Am Dent Assoc. 1983;107(3):420-4.

23- Opdebeeck H, Bell WH, Eisenfeld J, Mishelevich D. Comparative study between the SFS and LFS rotation as a possible morphogenic mechanism. Am J Orthod. 1978;74(5):509-21.

24- Õrmeno G, Miralles R, Santander H, Casassus R, Ferrer P, Palazzi C, et al. Body position effects on sternocleidomastoid and masseter emg pattern activity in patients undergoing occlusal splint therapy. Cranio. 1997;15(4):300-9.

25- Parker MW. A dynamic model of etiology in temporomandibular disorders. J Am Dent Assoc. 1990;120(3):283-90.

26- Peters RA, Gross SG. Clinical management of temporomandibular disorders and orofacial pain. Chicago: Quintessence; 1995. p. 123-60.

27- Price DD, McGrath PA, Rafii A, Buckingham B. The validation of visual analogue scales as ratio scale measures for chronic and experimental pain. Pain. 1983;17(1):45-56.

28- Santander H, Miralles R, Jimenez A, Zuñiga C, Rocabado M, Moya $\mathrm{H}$. Influence of stabilization occlusal splint on craniocervical relationships. Part II: Eelectromyographic analysis. Cranio. 1994;12(4):227-33.

29- Solow B, Tallgren A. Head posture and craniofacial morphology. Am J Phys Anthropol. 1976;44(3):417-35.

30- Wright EF, Domenech MA, Fischer JR Jr. Usefulness of posture training for patients with temporomandibular disorders. J Am Dent Assoc. 2000;131(2):202-10. 